\title{
Attractor solutions for general hessence dark energy
}

\author{
M. Alimohammadi* and H. Mohseni Sadjadi \\ School of Physics, University of Tehran, \\ North Karegar Ave., Tehran, Iran.
}

October 25, 2018

\begin{abstract}
As a candidate for the dark energy, the hessence model has been recently introduced. We discuss the critical points of this model in almost general case, that is for arbitrary hessence potential and almost arbitrary hessence-background matter interaction. It is shown that in all models, there always exist some stable late-time attractors. It is shown that our general results coincide with those solutions obtained earlier for special cases, but some of them are new. These new solutions have two unique characteristics. First the hessence field has finite value in these solutions and second, their stabilities depend on the second derivative of the hessence potential.
\end{abstract}

\section{Introduction}

In recent years, astronomical observations from type Ia supernova [1], WMAP data [2], and large scale structure surveys [3], have shown that the expansion of the universe is accelerated. Although there is no clear understanding of the mechanism leading to this acceleration, but it is believed that about $70 \%$ of the total energy density of universe consists of this unknown energy, i.e. dark energy, which leads to this expansion. The simplest explanation of dark energy is a cosmological constant $\Lambda$ of order $\left(10^{-3} \mathrm{ev}\right)^{4}$. Unfortunately it is about 120 orders smaller than the naive expectations, gives rise to the idea of a dynamical nature of this energy. The possible dynamical explanations have been introduced in different frameworks, such as quintessence [4], phantom [5], k-essence [6], tachyons [7], etc.

In studying the dark energy, the equation of state parameter $w_{\mathrm{de}}=p_{\mathrm{de}} / \rho_{\mathrm{de}}$ plays an important role, where $p_{\mathrm{de}}$ and $\rho_{\mathrm{de}}$ are the pressure and energy density of the dark energy, respectively. This parameter is always equal to constant -1 in cosmological constant model, but it can be a dynamical variable in the above mentioned dynamical models. This is an important point since the present data seems to slightly favor an evolving dark energy with $w_{\text {de }}$ being below -1 around present epoch, [8], from $w_{\mathrm{de}}>-1$ in the near past [9].

*alimohmd@ut.ac.ir 
To be definite, we consider the following action

$$
S=\int \mathrm{d}^{4} x \sqrt{-g}\left(-\frac{\mathcal{R}}{16 \pi G}+\mathcal{L}_{D E}+\mathcal{L}_{m}\right),
$$

where $g$ is the determinant of the metric $g_{\mu \nu}, \mathcal{R}$ is the Ricci scalar, $\mathcal{L}_{D E}$ and $\mathcal{L}_{m}$ are the Lagrangian densities of the dark energy and matter, respectively. In the case of quintessence, the Lagrangian density is

$$
\mathcal{L}_{\text {quintessence }}=\frac{1}{2}\left(\partial_{\mu} \phi\right)^{2}-V(\phi),
$$

where $\phi$ is a real scalar field. In a spatially flat Friedmann-Robertson-Walker (FRW) universe with homogeneous $\phi, w$ is

$$
w_{\text {quintessence }}=\frac{\dot{\phi}^{2} / 2-V(\phi)}{\dot{\phi}^{2} / 2+V(\phi)} \text {. }
$$

which results $-1 \leq w_{\text {quintessence. }}$ The Lagrangian density of phantom scalar field is

$$
\mathcal{L}_{\text {phantom }}=-\frac{1}{2}\left(\partial_{\mu} \phi\right)^{2}-V(\phi),
$$

from which

$$
w_{\text {phantom }}=\frac{-\dot{\phi}^{2} / 2-V(\phi)}{-\dot{\phi}^{2} / 2+V(\phi)},
$$

where for $\rho \geq 0$, which comes from $H^{2}=(8 \pi G / 3) \rho$, it results $w_{\text {phantom }} \leq-1$. So we can not cross the phantom-divide-line $w=-1$ in quintessence or phantom model alone. A possible way to overcome this problem is considering two real fields, which one behaves as quintessence and other one as phantom field. The resulting model, called the quintom model, has the following Lagrangian $[9,10]$

$$
\mathcal{L}_{\text {quintom }}=\frac{1}{2}\left(\partial_{\mu} \phi_{1}\right)^{2}-\frac{1}{2}\left(\partial_{\mu} \phi_{2}\right)^{2}-V\left(\phi_{1}, \phi_{2}\right),
$$

with

$$
w_{\text {quintom }}=\frac{{\dot{\phi_{1}}}^{2} / 2-{\dot{\phi_{2}}}^{2} / 2-V\left(\phi_{1}, \phi_{2}\right)}{{\dot{\phi_{1}}}^{2} / 2-{\dot{\phi_{2}}}^{2} / 2+V\left(\phi_{1}, \phi_{2}\right)} .
$$

Now it is obvious that $w_{\text {quintom }} \geq-1$ when ${\dot{\phi_{1}}}^{2} \geq{\dot{\phi_{2}}}^{2}$ and $w_{\text {quintom }}<-1$ when $\dot{\phi}_{1}{ }^{2}<\dot{\phi}_{2}{ }^{2}$. So crossing the phantom-divide-line is, in principle, possible in quintom model. See, for example, [10] and [11].

Instead of introducing two independent real scalar field to describe a quintom model, it is also natural to consider a single complex scalar field. The resulting spintessence model of dark energy [12-16], has the following Lagrangian density

$$
\mathcal{L}_{\text {spintessence }}=\frac{1}{2}\left(\partial^{\mu} \Phi^{*}\right)\left(\partial_{\mu} \Phi\right)-V(|\Phi|) .
$$

Using $\Phi=\phi_{1}+i \phi_{2}$, the kinetic term of eq. (8) reduces to the kinetic terms of eq.(6). Also the above Lagrangian is invariant under $\Phi \rightarrow e^{i \alpha} \Phi$ which leads to a conserved charge. Unfortunately this model suffers from the problem of Q-ball formation $[12,14]$. Q-ball is a kind of nontopological soliton which except in some 
special cases of spintessence with unnatural potentials, grows exponentially and depends on the potential, can be either stable at the late-time to be a dark matter, or decay into other particles. Therefore the spintessence model can not be a viable candidate for the dark energy.

To avoid the difficulty of Q-ball formation and also to introduce another possibility for mysterious dark energy problem, a non-canonical complex scalar field, called hessence, has been recently introduced in [17]. In the hessence model, the phantom-like role is played by the so called internal motion $\dot{\theta}$, where $\theta$ is the internal degree of freedom of hessence. There is a conserved charge $Q$ in this model which makes the physics of hessence more interesting, and the transition from $w_{h}>-1$ to $w_{h}<-1$ or vice versa is also possible. Another interesting feature of hessence model is that it is free of big-rip [18]. If $w<-1$ in an expanding FRW universe, then the positive energy density of a phantom matter generally becomes infinite in finite time, overcoming all other forms of matter and hence leads to the late-time singularity called the "big-rip" [19].

By considering two specific hessence potentials, i.e. the exponential and the (inverse) power law, and four different interaction forms between hessence and background perfect fluid, the late-time attractors of hessence model have been studied in [18]. In each case, different scaling and hessence-dominated solutions have been obtained and their stability properties have been studied.

In this paper we are going to study the late-time attractors of the almost general hessence model, with arbitrary hessence potential and almost arbitrary hessence-background matter interaction term. By almost arbitrary, we mean that the hessence potential and hessence-background matter interaction terms are arbitrary functions of dimensionless variables defined in (22). Specifically, we mean eqs. (33) and (34). We show that there always exist some stable solutions, in scaling or hessence-dominated form, which some of them have not been appeared in special cases studied in [18]. These new solutions have two interesting unique characteristics which are absent in other solutions. First their stability depends on the second derivative of the hessence potential, and second the hessence field has finite value in these solutions. The significance of the second derivative of the potential in the late-time behaviors has been also revealed for quintessence model in [20], in which some conditions have been imposed on the first and second derivatives of the potential.

The scheme of the paper is as follows. In section 2, we briefly introduce the main points of hessence model and the system of equations which determines the critical points in terms of dimensionless variables. In section 3, we consider the general hessence potential, but assuming no hessence-background matter interaction. It is shown that there exists five general solutions for critical points which three of them are stable under specific conditions. Finally in section 4 , the hessence potential and hessence-background matter interaction are considered arbitrary and it is shown that there are, in general, six classes of solutions for critical points. The stability of solutions is discussed in special cases. It is shown that all the solutions of [18] can be obtained from our general results.

We use the units $\hbar=c=1, \kappa^{2}=8 \pi G$ and adopt the metric convention as $(+,-,-,-)$ throughout the paper. 


\section{Hessence model}

Following [17], the hessence field introduced by a non-canonical complex scalar field

$$
\Phi=\phi_{1}+i \phi_{2}
$$

with Lagrangian density

$$
\mathcal{L}_{h}=\frac{1}{4}\left[\left(\partial_{\mu} \Phi\right)^{2}+\left(\partial_{\mu} \Phi^{*}\right)^{2}\right]-U\left(\Phi^{2}+\Phi^{* 2}\right)=\frac{1}{2}\left[\left(\partial_{\mu} \phi\right)^{2}-\phi^{2}\left(\partial_{\mu} \theta\right)^{2}\right]-V(\phi),
$$

where the new fields $(\phi, \theta)$ are defined through

$$
\phi_{1}=\phi \cosh \theta, \quad \phi_{2}=\phi \sinh \theta .
$$

In a spatially flat FRW universe with scale factor $a(t)$, the equations of motion for $\phi$ and $\theta$, when they are considered homogeneous, are

$$
\begin{aligned}
& \ddot{\phi}+3 H \dot{\phi}+\phi \dot{\theta}^{2}+V_{, \phi}=0, \\
& \phi^{2} \ddot{\theta}+\left(2 \phi \dot{\phi}+3 H \phi^{2}\right) \dot{\theta}=0,
\end{aligned}
$$

where $H \equiv \dot{a} / a$ is the Hubble parameter and overdot and subscript " , $\phi$ " denote the derivatives with respect to cosmic time $t$ and $\phi$, respectively. Eq. (13) implies

$$
Q=a^{3} \phi^{2} \dot{\theta}=\text { const., }
$$

where $Q$ is the total conserved charge due to the symmetry of Lagrangian (10) under the transformation $\phi \rightarrow \phi$ and $\theta \rightarrow \theta-i \alpha$. Substituting eq. (14) into (12), one has

$$
\ddot{\phi}+3 H \dot{\phi}+\frac{Q^{2}}{a^{6} \phi^{3}}+V_{, \phi}=0 .
$$

The pressure and energy density of hessence are

$$
p_{h}=\frac{1}{2} \dot{\phi}^{2}-\frac{Q^{2}}{2 a^{6} \phi^{2}}-V(\phi), \quad \rho_{h}=\frac{1}{2} \dot{\phi}^{2}-\frac{Q^{2}}{2 a^{6} \phi^{2}}+V(\phi) .
$$

The Friedmann equation and Raychaudhuri equation are given by, respectively,

$$
\begin{aligned}
& H^{2}=\frac{\kappa^{2}}{3}\left(\rho_{h}+\rho_{m}\right), \\
& \dot{H}=-\frac{\kappa^{2}}{2}\left(\rho_{h}+\rho_{m}+p_{h}+p_{m}\right),
\end{aligned}
$$

where $p_{m}$ and $\rho_{m}$ are the pressure and energy density of background matter, respectively. The background matter is described by a perfect fluid with barotropic equation of state

$$
p_{m}=w_{m} \rho_{m} \equiv(\gamma-1) \rho_{m},
$$

where $0<\gamma<2$. In particular, $\gamma=1$ and $4 / 3$ correspond to dust matter and radiation, respectively. 
To introduce the interaction between hessence and background matter, it is assumed that it can be described by an interaction term $C$ in the energy balance $[18,21]$

$$
\begin{gathered}
\dot{\rho}_{h}+3 H\left(\rho_{h}+p_{h}\right)=-C, \\
\dot{\rho}_{m}+3 H\left(\rho_{m}+p_{m}\right)=C,
\end{gathered}
$$

which preserves the total energy conservation equation $\dot{\rho}_{t o t}+3 H\left(\rho_{t o t}+p_{t o t}\right)=$ 0. $C=0$ corresponds to no interaction between hessence and background matter and when $C \neq 0$, a new term due to $C$ will appear in the right hand side of eq. (15).

Following [22] and many other papers, if we introduce the following dimensionless variables

$$
x \equiv \frac{\kappa \dot{\phi}}{\sqrt{6} H}, \quad y \equiv \frac{\kappa \sqrt{V}}{\sqrt{3} H}, \quad z \equiv \frac{\kappa \sqrt{\rho_{m}}}{\sqrt{3} H}, \quad u \equiv \frac{\sqrt{6}}{\kappa \phi}, \quad v \equiv \frac{\kappa}{\sqrt{6} H} \frac{Q}{a^{3} \phi},
$$

then using eqs.(16)-(18), (20), and (21), the evolution equations of these variables become

$$
\begin{aligned}
x^{\prime} & =3 x\left(x^{2}-v^{2}+\frac{\gamma}{2} z^{2}-1\right)-u v^{2}-\sqrt{\frac{3}{2}} y^{2} f-C_{1}, \\
y^{\prime} & =3 y\left(x^{2}-v^{2}+\frac{\gamma}{2} z^{2}\right)+\sqrt{\frac{3}{2}} x y f, \\
z^{\prime} & =3 z\left(x^{2}-v^{2}+\frac{\gamma}{2} z^{2}-\frac{\gamma}{2}\right)+C_{2}, \\
u^{\prime} & =-x u^{2}, \\
v^{\prime} & =3 v\left(x^{2}-v^{2}+\frac{\gamma}{2} z^{2}-1\right)-x u v .
\end{aligned}
$$

Prime denotes derivative with respect to the e-folding time $\mathcal{N} \equiv \ln a$, and

$$
\begin{gathered}
f \equiv \frac{V_{, \phi}}{\kappa V}, \\
C_{1} \equiv \frac{\kappa C}{\sqrt{6} H^{2} \dot{\phi}}, \quad C_{2} \equiv \frac{\kappa C}{2 \sqrt{3} H^{2} \sqrt{\rho_{m}}}=\frac{x}{z} C_{1} .
\end{gathered}
$$

The Friedmann equation (17) becomes

$$
x^{2}+y^{2}+z^{2}-v^{2}=1,
$$

and the fractional energy densities are

$$
\Omega_{h}=\frac{\rho_{h}}{\rho_{c}}=x^{2}+y^{2}-v^{2}, \quad \Omega_{m}=\frac{\rho_{m}}{\rho_{c}}=z^{2},
$$

where $\rho_{c}=\frac{3 H^{2}}{\kappa}$ is the critical energy density. The equation of states of hessence and whole system are

$$
w_{h}=\frac{p_{h}}{\rho_{h}}=\frac{x^{2}-v^{2}-y^{2}}{x^{2}-v^{2}+y^{2}}, \quad w_{e f f}=\frac{p_{h}+p_{m}}{\rho_{h}+\rho_{m}}=x^{2}-v^{2}-y^{2}+(\gamma-1) z^{2} .
$$

The critical points $(\bar{x}, \bar{y}, \bar{z}, \bar{u}, \bar{v})$ are obtained by imposing the conditions $\bar{x}^{\prime}=$ $\bar{y}^{\prime}=\bar{z}^{\prime}=\bar{u}^{\prime}=\bar{v}^{\prime}=0$. 
Note that we take

$$
f=f(u),
$$

and

$$
C_{1}=C_{1}(x, z, u), \quad C_{2}=C_{2}(x, z, u) .
$$

It is because $V=V(\phi)$, so $f$ is assumed to be a function of only one variable $u$. Also because of eq.(30), only four of the variables (22) are independent, which we can take them $x, y, z$, and $u$. But the dependence of hessence-background matter interaction to the potential $V$ (or variable $y$ ) is meaningless, so $C$ s are taken to be arbitrary functions of variables $x, z$ and $u$. Really eq. (33) does not constraint the potential $V$, but eq. (34) restricts the possible interaction term $C$. In this way the eqs. (23)-(27) become autonomous and we need not consider any further variables. For example $f$ is not and extra variable since $f^{\prime}=0$ leads to, for an arbitrary potential, $u^{\prime}=0$.

\section{Attractors in $C=0$ case}

To obtain the attractors for arbitrary hessence potential and when there is no hessence-background matter interaction, we must solve eq. (30) and the set of equations (23)-(27), when setting zero, in $C_{1}=C_{2}=0$. Eq.(26) results $\bar{u}=0$ or $\bar{x}=0$ and eq.(25) results $\bar{z}=0$ or $\bar{x}^{2}-\bar{v}^{2}+(\gamma / 2) \bar{z}^{2}-\gamma / 2=0$. So we have four possibilities: $\mathrm{I}=(\bar{u}=0, \bar{z}=0), \mathrm{II}=\left(\bar{u}=0, \bar{x}^{2}-\bar{v}^{2}+(\gamma / 2) \bar{z}^{2}-\gamma / 2=0\right)$, $\mathrm{III}=(\bar{x}=0, \bar{z}=0)$ and $\mathrm{IV}=\left(\bar{x}=0, \bar{x}^{2}-\bar{v}^{2}+(\gamma / 2) \bar{z}^{2}-\gamma / 2=0\right)$. In type $\mathrm{I}$ solution, eq.(27), using (30), reduces to $v y^{2}=0$, so it divides to $\mathrm{I} \cdot 1=(\bar{u}=0, \bar{z}=$ $0, \bar{v}=0)$ and I.2 $=(\bar{u}=0, \bar{z}=0, \bar{y}=0)$ solutions. The remaining variables can be easily found. The final results are represented in Table 1

\begin{tabular}{c|c|cccc}
\hline \hline Label & Critical Point $(\bar{x}, \bar{y}, \bar{z}, \bar{u}, \bar{v})$ & $\Omega_{h}$ & $\Omega_{m}$ & $w_{h}$ & $w_{\text {eff }}$ \\
\hline I.1 & $-\frac{\bar{f}}{\sqrt{6}}, \sqrt{1-\frac{\bar{f}^{2}}{6}}, 0,0,0$ & 1 & 0 & $-1+\frac{\bar{f}^{2}}{3}$ & $-1+\frac{\bar{f}^{2}}{3}$ \\
I.2 & $\bar{x}^{2} \geq 1,0,0,0, \pm \sqrt{\bar{x}^{2}-1}$ & 1 & 0 & 1 & 1 \\
II & $-\sqrt{\frac{3}{2}} \frac{\gamma}{f}, \sqrt{\frac{3 \gamma}{f^{2}}\left(1-\frac{\gamma}{2}\right)}, \sqrt{1-\frac{3 \gamma}{f^{2}}}, 0,0$ & $\frac{3 \gamma}{f^{2}}$ & $1-\frac{3 \gamma}{f^{2}}$ & $-1+\gamma$ & $-1+\gamma$ \\
III & $0,1,0, \bar{f}=0,0$ & 1 & 0 & -1 & -1 \\
IV & $0,0,1$, any, 0 & 0 & 1 & any & $-1+\gamma$ \\
\hline
\end{tabular}

Table 1: Critical points for arbitrary hessence potential when there is no hessence-background matter interaction.

The solutions with $\Omega_{h}=1$, i.e. solutions I.1, I.2 and, III, are hessencedominated, solution IV is background-matter-dominated and solution II is scaling solution. In solution III, $\bar{u}$ must be found by solving $\bar{f} \equiv f(\bar{u})=0$. Note that in all cases, $w_{\text {eff }}>-1$. It is also interesting that for potentials where $f^{2}(\bar{u}=0)=3 \gamma$, the solutions I.1 and II become degenerate.

In examples considered in [18], the potentials are

$$
\begin{aligned}
& V_{1}=V_{0} e^{-\lambda \kappa \phi}, \\
& V_{2}=V_{0}(\kappa \phi)^{n},
\end{aligned}
$$


or, using (28),

$$
\begin{aligned}
& f_{1}=-\lambda, \\
& f_{2}=\frac{n}{\kappa \phi}=\frac{n u}{\sqrt{6}} .
\end{aligned}
$$

It can be easily checked that our solutions (I.1, I.2, II and IV) and (I.1, I.2, and IV) reduce to those obtained in [18] for $V_{1}$ and $V_{2}$, respectively. The solution II does not exist for potential $V_{2}$ since $f_{2}(\bar{u}=0)=0$. The solution III is a new solution which has not been appeared in [18]. This is because the equation $\bar{f}=0$ results $\lambda=0$ for $V_{1}$, which is not acceptable, and results $\bar{u}=0$ for $V_{2}$, which reduces solution III to I.1.

To study the stability of the critical points I.1-IV, we must consider a small perturbation about the critical point $(\bar{x}, \bar{y}, \bar{z}, \bar{u}, \bar{v}): x \rightarrow \bar{x}+\delta x, y \rightarrow \bar{y}+\delta y$, $z \rightarrow \bar{z}+\delta z, u \rightarrow \bar{u}+\delta u$, and $v \rightarrow \bar{v}+\delta v$, in eqs.(23)-(26) with $C_{1}=C_{2}=0$, which due to Friedmann constraint (30), only four of them are independent. In this way one can found a $4 \times 4$ matrix $M$ defined through

$$
\frac{\mathrm{d}}{\mathrm{d} \mathcal{N}}\left(\begin{array}{c}
\delta q_{1} \\
\delta q_{2} \\
\delta q_{3} \\
\delta q_{4}
\end{array}\right)=M\left(\begin{array}{c}
\delta q_{1} \\
\delta q_{2} \\
\delta q_{3} \\
\delta q_{4}
\end{array}\right),
$$

where $\left(q_{1}, \cdots, q_{4}\right)$ are four chosen independent variables. The critical solutions are stable if the real part of all the eigenvalues of matrix $M$ are negative. The eigenvalues of matrix $M$ for our solutions are as following:

$$
\begin{aligned}
& \text { I. } 1:\left(-3 \bar{y}^{2},-6 \bar{y}^{2}, \frac{1}{2}\left(\bar{f}^{2}-3 \gamma\right), 0\right), \\
& \text { I. } 2:\left(0,0, \frac{3}{2}(2-\gamma), 3+\sqrt{\frac{3}{2}} \bar{x} \bar{f}\right), \\
& \text { III }:\left(-6,-\frac{3 \gamma}{2} \quad, \quad-\frac{3}{2}+\frac{\sqrt{9+2 \sqrt{6} \bar{u}^{2} \bar{f}^{\prime}}}{2},-\frac{3}{2}-\frac{\sqrt{9+2 \sqrt{6} \bar{u}^{2} \bar{f}^{\prime}}}{2}\right), \\
& \text { IV }:\left(0, \frac{3 \gamma}{2}, 3(\gamma-2), \frac{3}{2}(\gamma-2)\right),
\end{aligned}
$$

in which $\left(q_{1}, \cdots, q_{4}\right)=(x, y, z, u)$ and $\bar{f}^{\prime}=(\mathrm{d} f / \mathrm{d} u)_{\bar{u}}$. It is clear that I.1 solution is stable if

$$
\bar{f}^{2} \leq 3 \gamma
$$

I.2 solution is unstable since $\gamma<2$, III solution is stable if

$$
\left(\frac{\mathrm{d} f}{\mathrm{~d} u}\right)_{\bar{u}} \leq 0
$$

and IV is an unstable solution since $\gamma>0$.

For solution II, it is easier to use $\left(q_{1}, \cdots, q_{4}\right)=(x, z, u, v)$. Then it can be easily found that $l_{1}=0, l_{2}=\frac{3}{2}(\gamma-2)$, and $l_{3}$ and $l_{4}$ are roots of equation $l^{2}+b l+c=0$ with

$$
b=\frac{3}{2}(\gamma-2)
$$




$$
c=\frac{9 \gamma}{2 \bar{f}^{2}}(2-\gamma)\left(\bar{f}^{2}-3 \gamma\right) .
$$

$l_{3}$ and $l_{4}$ are non-positive if $b \geq 0$ and $c \geq 0$. As $\gamma<2$, the solution II is stable if

$$
\bar{f}^{2} \geq 3 \gamma
$$

So for any potential $V$, there always exists at least one stable attractor. A hessence-dominated attractor (I.1) if $\bar{f}^{2} \leq 3 \gamma$ or a scaling attractor (II) if $\bar{f}^{2} \geq$ $3 \gamma$.

It is interesting to note that the solution III has two unique properties. First, it is the only stable attractor which has the non-vanishing $\bar{u}$ value, i.e. finite value of hessence field $\bar{\phi}$. Second, it is the only attractor which its stability depends on the derivative of $f$ (eq. (45)). In other words, the stable attractors I.1 and II can not distinguish between different potentials with the same $\bar{f}$ value, but the attractor III does.

As examples of potentials which have the solution III as a stable attractor, we may consider $V_{3}=V_{0} \sin (\kappa \phi / \sqrt{6})$ and $V_{4}=V_{0} \cos (\kappa \phi / \sqrt{6})$. For $V_{3}$, we have $f_{3}=-\left(1 / \kappa u^{2}\right) \cot (1 / u)$, which results $\bar{u}=[(2 n+1) \pi / 2]^{-1}$, with $n=0,1,2, \cdots$, as the solution of $\bar{f}_{3}=0$ equation. It really has infinite number of attractors. Then $(\mathrm{d} f / \mathrm{d} u)_{\bar{u}}=-(1 / \kappa)[(2 n+1) \pi / 2]^{4}<0$, which shows that the attractors are stable. The same is true for $V_{4}$ potential.

\section{Attractors in the presence of hessence-background matter interaction}

In this case, we must solve eq.(30) and the set of equations (23)-(27), when setting zero, for arbitrary $C$ function. Eq.(26) results $\bar{u}=0$ or $\bar{x}=0$. In each case, we consider eight cases in which each of the variables $\bar{y}, \bar{z}$ and $\bar{v}$ has two possibilities, zero and not zero, and then check the consistency of the equations. The final results are as following:

$$
\text { solution } 1:\left\{\bar{x}=-\frac{\bar{f}}{\sqrt{6}}, \bar{y}=\sqrt{1-\frac{\bar{f}^{2}}{6}}, \bar{z}=\bar{u}=\bar{v}=0, \bar{C}_{1}=\bar{C}_{2}=0\right\} .
$$

Note that the equations $\bar{C}_{1}=\bar{C}_{2}=0$ imply that the functional form of $C_{1}$ and $C_{2}$ must be such that they are identically equal to zero at this critical point, otherwise this solution does not exist.

$$
\text { solution } 2:\left\{\bar{x}^{2} \geq 1, \bar{y}=\bar{z}=\bar{u}=0, \bar{v}= \pm \sqrt{\bar{x}^{2}-1}, \bar{C}_{1}=\bar{C}_{2}=0\right\} .
$$

In this case, the equations $\bar{C}_{1}=\bar{C}_{2}=0$ can generally determine the allowed value of $\bar{x}$. If $\bar{C}_{1}$ and $\bar{C}_{2}$ are identically equal to zero at $\bar{y}=\bar{z}=\bar{u}=0$, as they are in $C=0$ case, then $\bar{x}$ can choose any arbitrary value.

$$
\text { solution } 3:\left\{\bar{x}=\bar{y}=0, \bar{z}=1, \bar{u}, \bar{v}=0, \bar{C}_{1}=\bar{C}_{2}=0\right\} \text {. }
$$

The value of $\bar{u}$ is generally determined by solving $\bar{C}_{1}=\bar{C}_{2}=0$.

$$
\text { solution } 4:\left\{\bar{x}=0, \bar{y}=1, \bar{z}=0, \bar{u}, \bar{v}=0, \bar{C}_{1}=-\sqrt{\frac{3}{2}} \bar{f}, \bar{C}_{2}=0\right\} \text {, }
$$


where the last two equations can generally determine $\bar{u}$.

solution $5:\left\{\bar{y}=\bar{u}=\bar{v}=0, \bar{C}_{1}=3 \bar{x}\left(\bar{x}^{2}+\frac{\gamma}{2} \bar{z}^{2}-1\right), \bar{C}_{2}=-3 \bar{z}\left(\bar{x}^{2}+\frac{\gamma}{2} \bar{z}^{2}-\frac{\gamma}{2}\right), \bar{x}^{2}+\bar{z}^{2}=1\right\}$.

$\bar{x}$ and $\bar{z}$ are found by solving the above equations. The last solution is:

solution $6: \quad\left\{\quad \bar{y}=\sqrt{1-\bar{x}^{2}-\bar{z}^{2}}, \bar{u}=\bar{v}=0, \bar{C}_{1}=3 \bar{x}\left(\bar{x}^{2}+\frac{\gamma}{2} \bar{z}^{2}-1\right)-\sqrt{\frac{3}{2}} \bar{y}^{2} \bar{f}\right.$,

$$
\left.\bar{C}_{2}=-3 \bar{z}\left(\bar{x}^{2}+\frac{\gamma}{2} \bar{z}^{2}-\frac{\gamma}{2}\right), 3\left(\bar{x}^{2}+\frac{\gamma}{2} \bar{z}^{2}\right)+\sqrt{\frac{3}{2}} \bar{x} \bar{f}=0\right\} .
$$

At $C=0$, solutions $1,2,3,4$, and 6 reduce to I.1, I.2, IV, III, and II of Table1 respectively. The solutions 1,2 , and 4 are hessence-dominated, solution 3 is background-matter-dominated and 5 and 6 are generally scaling solutions. Among these solutions, there are only two solutions 3 and 4 in which $\bar{u}$ can principally be different from zero, which have not been appeared in examples discussed in [18].

In [18], besides the non-interacting $C=0$ case, three following interactions have been considered:

$$
\begin{aligned}
C_{1}^{(\mathrm{II})} & =\sqrt{\frac{3}{2}} \alpha z^{2}, \\
C_{1}^{(\mathrm{III})} & =\frac{3}{2} \frac{\beta}{x}, \\
C_{1}^{(\mathrm{IV})} & =\frac{3}{2} \eta \frac{z^{2}}{x},
\end{aligned}
$$

in which $\alpha, \beta$ and $\eta$ are some constants. In both of the solutions 3 and $4, \bar{x}$ is zero, so $C_{1}^{(\mathrm{III})}$ diverges and $C_{1}^{(\mathrm{IV})}$ is not generally well-defined and therefore these critical points do not exist in these cases. So we only consider the $C_{1}^{(\text {II })}$ case. For $V=V_{1}$ potential, with $f_{1}=-l$, solution 3 does not exist since $\bar{C}_{1}=0$ leads to $\alpha=0$ which is not acceptable, and solution 4 does also not exist as $\bar{C}_{1}=-\sqrt{\frac{3}{2}} \bar{f}$ results in $l=0$ which again is not acceptable. For $V=V_{2}$ potential, with $f_{2}=n u / \sqrt{6}$, solution 3 leads to $\alpha=0$ which is not acceptable, and solution 4 results in $\bar{u}=0$, which does not lead to a $\bar{u} \neq 0$ solution.

It may be useful to reproduce all the critical points of at least one of the cases studied in [18] in more detail. We consider, as an example, $V=V_{1}$ and $C=C_{1}^{(\mathrm{II})}$. So

$$
f=-l, \quad C_{1}=\sqrt{\frac{3}{2}} \alpha z^{2}, \quad C_{2}=\sqrt{\frac{3}{2}} \alpha x z .
$$


The solutions 1-6 result in:

solution $1:\left\{\bar{x}=\frac{l}{\sqrt{6}}, \bar{y}=\sqrt{1-\frac{l^{2}}{6}}, \bar{z}=\bar{u}=\bar{v}=0\right\}$,

solution $2:\left\{\bar{x}^{2} \geq 1, \bar{y}=\bar{z}=\bar{u}=0, \bar{v}= \pm \sqrt{\bar{x}^{2}-1}\right\}$,

solution $3: \bar{C}_{1}=0 \rightarrow \alpha=0$ (is not acceptable),

solution $4: \quad \bar{C}_{1}=-\sqrt{\frac{3}{2}} \bar{f} \rightarrow l=0$ (is not acceptable),

solution $5:\left\{\bar{x}=\sqrt{\frac{2}{3}} \frac{\alpha}{\gamma-2}, \bar{y}=0, \bar{z}=\sqrt{1-\frac{2 \alpha^{2}}{3(\gamma-2)^{2}}}, \bar{u}=\bar{v}=0\right\}$,

solution $6:\left\{\bar{x}=\sqrt{\frac{3}{2}} \frac{\gamma}{l+\alpha}, \bar{y}=\sqrt{\frac{2 \alpha^{2}-3(\gamma-2) \gamma+2 \alpha l}{2(\alpha+l)^{2}}}, \bar{z}=\sqrt{\frac{l(l+\alpha)-3 \gamma}{(l+\alpha)^{2}}}, \bar{u}=\bar{v}=0\right\}$,

which coincide with those in Table II of [18]. Note that in that table, four of the solutions ( $2 \mathrm{p}, 2 \mathrm{~m}, 4$ and 5 ) are not independent solutions and are special cases of the first solution.

The stability studies of these critical points depends on the precise value of the function $C$. But it may be interesting to study the conditions under which the derivative of $f$ becomes important in the stability properties of the critical points. Consider the most general case $C_{1}=C_{1}(x, z, u)$. It can be shown that the coefficient of $\bar{f}^{\prime}$ term in equation $\operatorname{det}(M-\hat{1} l)=0$, where $\hat{1}$ stands for $4 \times 4$ unit matrix, is

$$
\frac{\bar{y}^{2} \bar{u}^{2}}{\bar{z}^{2}} h(\bar{x}, \bar{y}, \bar{z}, \bar{u})
$$

which shows that the derivative of $f$ survives only if

$$
\bar{y} \neq 0 \text { and } \bar{u} \neq 0 \text {. }
$$

Therefore the solution 4 is the only one in which we expect $\bar{f}^{\prime}$ may be important. Focusing on this solution, the eigenvalue problem is still very complicated in general case and it is better to consider the more restricted cases. As an example, we consider the potentials and $C$ 's in which $\bar{f}=\overline{\left(C_{1} / z\right)}=\overline{\left(\partial C_{1} / \partial x\right)}=0$. Under these conditions, the equation $\operatorname{det}(M-\hat{1} l)=0$ leads to

$$
l^{4}+a_{1} l^{3}+a_{2} l^{2}+a_{3} l+a_{4}=0,
$$

where

$$
\begin{aligned}
& a_{1}=9+\frac{3}{2} \gamma, \\
& a_{2}=18+\frac{27}{2} \gamma-\bar{u}^{2}\left(\bar{C}_{1, u}+\sqrt{\frac{3}{2}} \bar{f}^{\prime}\right), \\
& a_{3}=-3 \bar{u}^{2}\left(\bar{C}_{1, u}+\sqrt{\frac{3}{2}} \bar{f}^{\prime}\right)\left(2+\frac{\gamma}{2}\right)+27 \gamma, \\
& a_{4}=-9 \gamma \bar{u}^{2}\left(\bar{C}_{1, u}+\sqrt{\frac{3}{2}} \bar{f}^{\prime}\right),
\end{aligned}
$$

where $\bar{C}_{1, u}=\overline{\left(\partial C_{1} / \partial u\right)}$. Now it is well known that $\sum_{i} l_{i}=-a_{1}, \sum_{i \neq j} l_{i} l_{j}=a_{2}$, $\sum_{i \neq j \neq k} l_{i} l_{j} l_{k}=-a_{3}$, and $l_{1} l_{2} l_{3} l_{4}=a_{4}$, where $l_{1} \cdots l_{4}$ are the roots of eq. (64). 
So all the roots are non-positive only if $a_{i} \geq 0(i=1, \cdots, 4)$, which provided if

$$
\bar{f}^{\prime} \leq-\sqrt{\frac{2}{3}} \bar{C}_{1, u}
$$

In $C=0$, this condition of stability reduces to eq. (45).

\section{Conclusion}

In this paper, we study the attractor solutions of the general hessence model by studying the 4-dimensional phase space of the theory. The hessence model is a non-canonical complex scalar theory which can be a candidate of dark energy with some interesting properties, among them is the possibility of crossing the $w=-1$ line. Comparing the Lagrangian of hessence model with quintom model shows that the $\theta$-( or equally charge $Q$-)term plays the role of the phantom field. In $Q=0$, the model reduced to quintessence model with no $w=-1$ crossing.

We consider an arbitrary hessence potential $V(\phi)$ and almost arbitrary hessence-background matter interaction term $C$, and find several results. We show that in $C=0$, there is always at least one stable attractor which depends on the value $\bar{f}=f(\bar{u}=0)$ : For $\bar{f}^{2} \leq 3 \gamma$ the hessence-dominated attractor I.1 and for $\bar{f}^{2} \geq 3 \gamma$ the scaling attractor II of Table 1. In all the attractor solutions we have $\bar{\phi} \rightarrow \infty$, except for the attractor III where $\bar{\phi}$ is finite and also its stability depends on the derivative $(d f / d u)_{\bar{u}}$. This kind of attractor did not appear in the previously studied cases and can be seen in potentials like $\sin (\kappa \phi) . \bar{v}$ is zero in all the stable attractors of Table 1 , but for arbitrary $C$-term, this is not the case.

For general $C$, we show that there generally exist six classes of attractor, which all of them can be stable in special cases ( for example $V=V_{1}$ and $C_{1}=C_{1}^{(I I)}$ of [18]). Among these solutions, solution 2 is the only one with property $\bar{v} \neq 0$. The solutions 3 and 4 have finite $\bar{\phi}$ value and the the solution 4 is the only one which its stability depends on $(d f / d u)_{\bar{u}}$, the features that can not be seen in the previously studied potentials.

Acknowledgement: We would like to thank the research council of the University of Tehran for partial financial support.

\section{References}

[1] A. G. Riess et al., Astron. J. 116 (1998) 1009; S. Perlmutter et al., Nature 391 (1998) 51; A. G. Riess et al., Astron. J. 118 (1999) 2668; S. Perlmutter et al., Astrophys. J. 517 (1999) 565; A. G. Riess et al., Astrophys. J. 536 (2000) 62; A. G. Riess et al., Astrophys. J. 560 (2001) 49.

[2] C. Bennet et al., Astrophys. J. (Suppl.) 148 (2003) 1; G. Hinshaw et al., Astrophys. J. (Suppl.) 148 (2003) 135; A. Kogut et al., Astrophys. J. (Suppl.) 148 (2003) 161; S. Bridle et al., Science 239 (2003) 1532; D. N. Spergel et al., Astrophys. J. (Suppl.) 148 (2003) 175; L. Page et al., Astrophys. J. (Suppl.) 148 (2003) 233.

[3] S. W. Allen et al., Mon. R. Astr. Soc. 535 (2004) 4571. 
[4] Y. Fujii, Phys. Rev. D26 (1982) 2580; L. H. Ford, Phys. Rev. D35 (1987) 2339; C. Wetterich, Nucl. Phys. B302 (1988) 668; B. Ratra and J. Peebles, Phys. Rev. D37 (1988) 321; Y. Fujii and T. Nishioka, Phys. Rev. D42 (1990) 361; E. J. Copeland, A. R. Liddle, and D. Wands, Ann. N. Y. Acad. Sci. 688(1993) 647; R. R. Caldwell, R. Dave and P. J. Steinhardt, Phys. Rev. Lett. 80 (1998) 1582; I. Zlatev, L. M. Wang and P. J. Steinhardt, Phys. Rev. Lett. 82 (1999) 896; P. J. Steinhardt, L. M. Wang and I. Zlatev, Phys. Rev. D59 (1999) 123504; D. F. Mota and C. van de Bruck, Astron. Astrophys. 421 (2004) 71.

[5] R. R. Caldwell, Phys. Lett. B545 (2002) 23; R. R. Caldwell, M. Kamionkowski and N. N. Weinberg, Phys. Rev. Lett. 91 (2003) 071301; S. M. Carroll, M. Hoffman and M. Trodden, Phys. Rev. D68 (2003) 023509; P. Singh, M. Sami and N. Dadhich, Phys. Rev. D68 (2003) 023522; S. Nojiri and S. D. Odintsov, Phys. Lett. B562 (2003) 147.

[6] C. Armendariz-Picon, V. Mukhanov and P. J. Steinhardt, Phys. Rev. Lett. 85 (2000) 4438; C. Armendariz-Picon, V. Mukhanov and P. J. Steinhardt, Phys. Rev. D63 (2001) 103510; T. Chiba, T. Okabe and M. Yamaguchi, Phys. Rev. D62 (2000) 023511; M. Malquarti, E. J. Copeland and A. R. Liddle, Phys. Rev. D68 (2003) 023512; M. Malquarti, E. J. Copeland, A. R. Liddle and M. Trodden, Phys. Rev. D67 (2003) 123503.

[7] G. W. Gibbons, Phys. Lett. B537 (2002) 1; T. Padmanabhan, Phys. Rev. D66 (2002) 021301; J. S. Bagla, H. K. Jassal and T. Padmanabhan, Phys. Rev. D67 (2003) 063504.

[8] D. Huterer and A. Cooray, Phys. Rev. D71 (2005) 023506; U. Alam, V. Sahni and A. A. Starobinsky, JCAP 0406 (2004) 008 ; Y. Wang and M. Tegmark, Phys. Rev. D71 (2005) 103513; R. Lazkoz, S. Nesseris and L. Perivolaropoulos, astro-ph/0503230.

[9] B. Feng, X. L. Wang and X. M. Zhang, Phys. Lett. B607 (2005) 35.

[10] Z. K. Guo, Y. S. Piao, X. M. Zhang and Y. Z. Zhang, Phys. Lett. B608 (2005) 177.

[11] X. F. Zhang, H. Li, Y. S. Piao and X. M. Zhang, astro-ph/0501652

[12] L. A. Boyle, R. R. Caldwell and M. Kamionkowski, Phys. Lett. B545 (2002) 17.

[13] J. A. Gu and W. Y. Hwang, Phys. Lett. B517 (2001) 1.

[14] S. Kasuya, Phys. Lett. B515 (2001) 121.

[15] X. Z. Li, J. G. Hao and D. J. Liu, Class. Quant. Grav. 19 (2002) 6049.

[16] S. Capozziello, S. Nojiri and S. D. Odintsov, Phys. Lett B632 (2006) 597.

[17] H. Wei, R. G. Cai and D. F. Zeng, Class. Quant. Grav. 22 (2005) 3189.

[18] H. Wei and R. G. Cai, Phys. Rev. D72 (2005) 123507. 
[19] R. R. Caldwell, M. Kamionkowski and N. N. Weinberg, Phys. Rev. Lett. 91 (2003) 071301.

[20] A. D. Rendall, Class. Quant. Grav. 22 (2005) 1655.

[21] W. Zimdahl and D. Pavon, Phys. Lett. B521 (2001) 133; R. G. Cai and A. Wang, JCAP 0503 (2005) 002; H. Wei and R. G. Cai, Phys. Rev. D71 (2005) 043504.

[22] E. J. Copeland, A. R. Liddle and D. Wands, Phys. Rev. D57 (1998) 4686; L. Amendola, Phys. Rev. D60 (1999) 043501; X. Z. Li, J. G. Hao and D. J. Liu, Class. Quant. Grav. 19 (2002) 6049; L. Amendola, C. Quercellini, D. Tocchini-Valentini and A. Pasqui, Astrophys. J. 583 (2003) L53. 